\title{
Two Feedback System Schemes for the Collider and Resistive Wall Instability
}

\author{
G. López \\ Superconducting Super Collider Laboratory* \\ 2550 Beckleymeade Avenue \\ Dallas, Texas 75237
}

October 1993

"Operated by the Universities Research Association, Inc., for the U.S. Department of Energy under Contract No. DE-AC35-89ER40486. 


\title{
Two Feedback System Schemes for the Collider and Resistive Wall Instability
}

\author{
G. López
}

\begin{abstract}
Two feedback systems proposed for the SSC Collider are studied from the point of view of the capability for controlling the resistive wall instability. The previous kick subtraction feedback scheme can damp the dipole mode resistive wall instability. However, the feedback system with digital filter processing cannot control this instability.
\end{abstract}




\subsection{INTRODUCTION}

Controlling the Dipole Mode Multibunch Instability (DMMI) due to the resistive wall impedance is of fundamental importance for the Collider to be operational. The growth time of the fundamental mode (lowest frequency) is estimated between $50 \mathrm{msec}$ and $150 \mathrm{msec},{ }^{1}$ depending on the beam tube and copper coated thickness, fractional part of the tune, and beam tube aperture. This time is quite short, so a reliable feedback system must be used to control this instability. A novel two-BPM, two-kicker feedback system was proposed originally in Reference 1 in order to have better control of this instability. The optimization of system parameters was already given. ${ }^{2-4}$ However, one would think that changing the correction scheme from a single kicker feedback system would result in a good control of this instability. There are two basic schemes proposed. ${ }^{5}$ One is the subtraction of the previous correction kick to the new one. The other scheme is using a digital filter processing with a more complicated correction kick. Using the computer code TADIMII (see Reference 1), the simulation of the resistive wall instability is carried out using these two schemes.

\subsection{RESISTIVE WALL AND PREVIOUS CORRECTION SUBTRACTION SCHEME}

The resistive wall impedance is treated as a resonant impedance, according to the approximation made in Reference 1 . The Collider is assumed to be a perfect linear machine having a uniform stainless steel beam pipe (1 mm thick, $4.6 \mathrm{~cm}$ aperture) coated with copper $\left(0.1 \mathrm{~mm}\right.$ thick). The fractional part of the tune $\left(1+\Delta_{i 3}\right)$ is assumed to be 0.777 . Therefore, the fundamental instability mode has an angular frequency $\left(\omega=\left|\Delta_{\beta}\right| \omega_{0}\right)$ of about $4.9 \mathrm{kHz}$, and the resonant impedance $\left(R_{\perp}\right)$ is about $1.6 \mathrm{G} \Omega / \mathrm{m}$. Figure 1 shows the relative separation of the Beam Position Monitor (BPM), kicker ( $K$ ), and Resonant Impedance $(R)$ in the ideal ring. The BPM and kicker have their coordinates according with their location at the Collider West Utility Region. The Courant-Snyder map is used to transport each of the 17424 bunches from element to element. The wake field felt by each bunch is that one generated at the resonant element ( $\mathrm{K}$ ). The correction kick, at the curn "n", provided by the kicker is given by"

$$
U_{n}=\frac{g}{\sqrt{\beta_{1} \beta_{2}}}\left(X_{n}-X_{n-1}+\delta X\right)
$$

where $g$ is the gain of the feedback system, $\beta_{1}$ and $\beta_{2}$ are the beta function at the location of the BPM and kicker, $\delta X$ represents the BPM resolution (noise), and $X_{n-1}$ represents the previous correction amplitude. The machine has a tune $\mu$ and the phase advance between the kicker and the resonator $(R)$ is $\phi$. The result of the simulations can be seen in Figure 2 where the average amplitude value at the BPM location is plotted as a function of the 
number of turns. Figure 3 shows the behavior of a single particular bunch. The gain (g), the flat-top time duration (Ft, in turns), the bandwidth (BW), and the BPM resolution $(\delta X)$ used are also shown in these figures.

This correction scheme works much better than the traditional one $\left(X_{n-1}=0\right)$ although it has a slight complication in recording the previous turn amplitude. However, this scheme cannot, even in principle, kill the instability in the same turn when the gain is equal to one. Such a thing might be desirable to control the emittance growth due the decoherence of the beam (see References 2, 3, and 4). Although the damping of the fundamental mode has been shown here, this novel feedback scheme can also damp the next few resistive instability modes $(k \leq 5)$. The higher modes are Landau dampened or controlled by a bunch-bybunch damper system. It is pointed out that, in fact, this scheme involves another BPM which is used in conjunction with the preceding one mainly for tune correction.

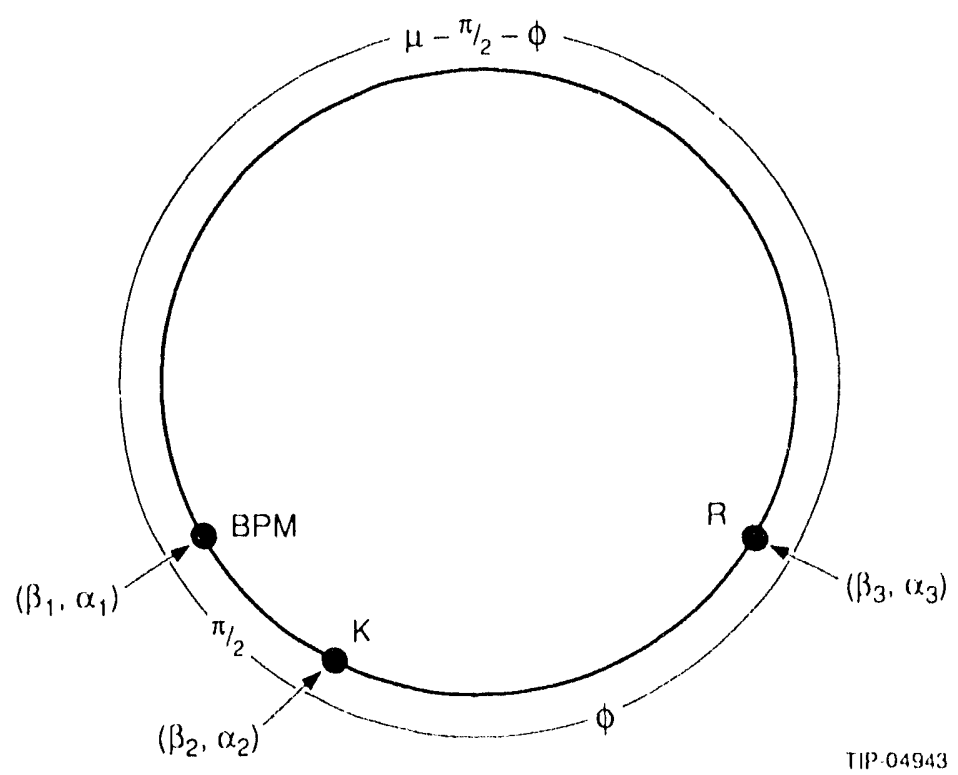

Figure 1. Collider model for the first scheme. 




Figure 2. Average displacement seen by the BPM

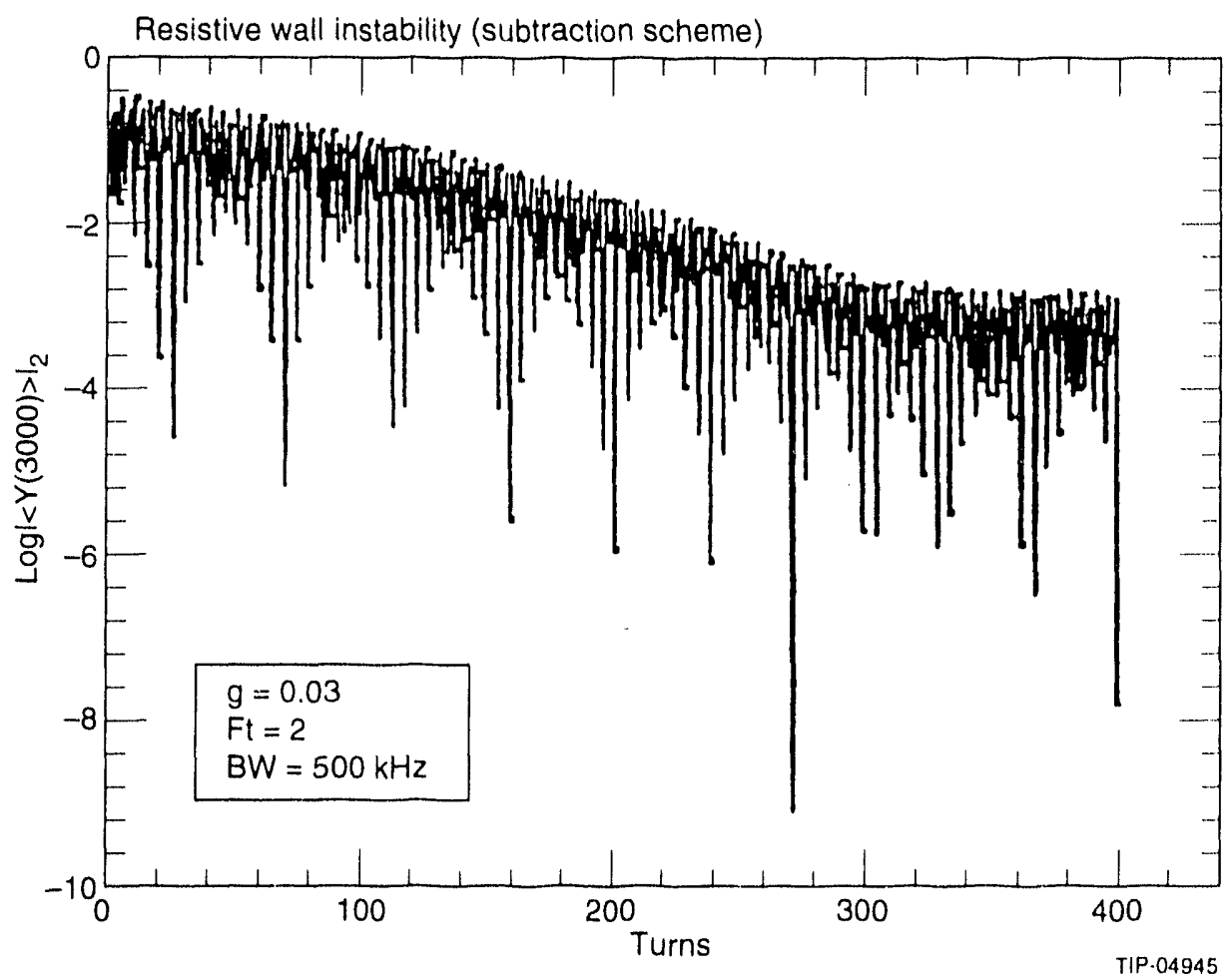

Figure 3. Behavior of bunch number 3000 . 


\subsection{DIGITAL FILTER PROCESSING FEEDBACK}

The idea of this novel feedback system is to use the information of two BPMs and one correction kicker. The BPMs and kicker have coordinates consisient with their location at the Collider West Utility. The configuration is shown in Figure 4. The transport approach of the bunches is the same as before.

The digital filter processing provides finite impulse response and finite memory. The correction kick is given by ${ }^{6}$

$$
U_{n}=U_{n-1}\left(1-\frac{1}{M}\right)+\frac{g \tilde{U}_{n}}{M}
$$

where $g$ is the gain of the feedback system, $U_{n-1}$ is the previous correction recorded, and $M$ is the sample of bunches taken which is given by the time average taken (which, in turn, defines the flat-top kicker duration time). $\widetilde{U}_{n}$ is a function depending on the tune $(\mu)$ and the information from the two BPM*

$$
\tilde{U}_{n}=-\Delta X_{n}^{\prime}-\frac{\Delta X_{n}}{\beta_{2}} \frac{\cos (\mu)}{\sin (\mu)}+\frac{\Delta X_{n-1}}{\beta_{2} \sin (\mu)}
$$

where $\Delta X_{n}$ and $\Delta X_{n}^{\prime}$ are given in terms of the tune, bunch coordinates at the BP.I2 $\left(X_{n}, X_{n}^{\prime}\right)$, and the previous correction $U_{n-1}$ as

$$
\Delta X_{n}=X_{n}-\left(1-g_{a}\right)\left(\cos (\mu) X_{n-1}+\beta_{2} \sin (\mu) X_{n-1}^{\prime}+\beta_{2} \sin (\mu) U_{n-1}\right)
$$

and

$$
\Delta X_{n}^{-1}=X_{n}^{-1}-\left(1-g_{n}\right)\left(-\frac{\sin (\mu) X_{n-1}}{\beta_{2}}+\cos (\mu) X_{n-1}^{-1}+\cos (\mu) U_{n-1}\right)+\delta X_{n}
$$

The parameter $g_{a}$ is chosen different from zero to damp the coherent oscillations motion. The average value of the bunch displacement as seen by BPM2 is shown in Figure 5. As Figure 5 shows, an initial damping is present, but, after some turns, the instability cannot be controlled, and the beam becomes unstable. This is seen more explicitly with the behavior of two different bunches shown in Figures 6 and 7 . This behavior secms to be caused by the very weak dependence of the correction kick on the amplitude of the coherent oscillations, as seen in Figure 8. The analysis was made with different gain (g), flat-top duration, $g_{a}$ values, and feedback noise. The result was the same, i.e., this fecdback scheme cannot control the resistive wall instability in the Collider.

* The original expressions did not contain the Courant-Snyder parameters since the coordinates were normalized. 


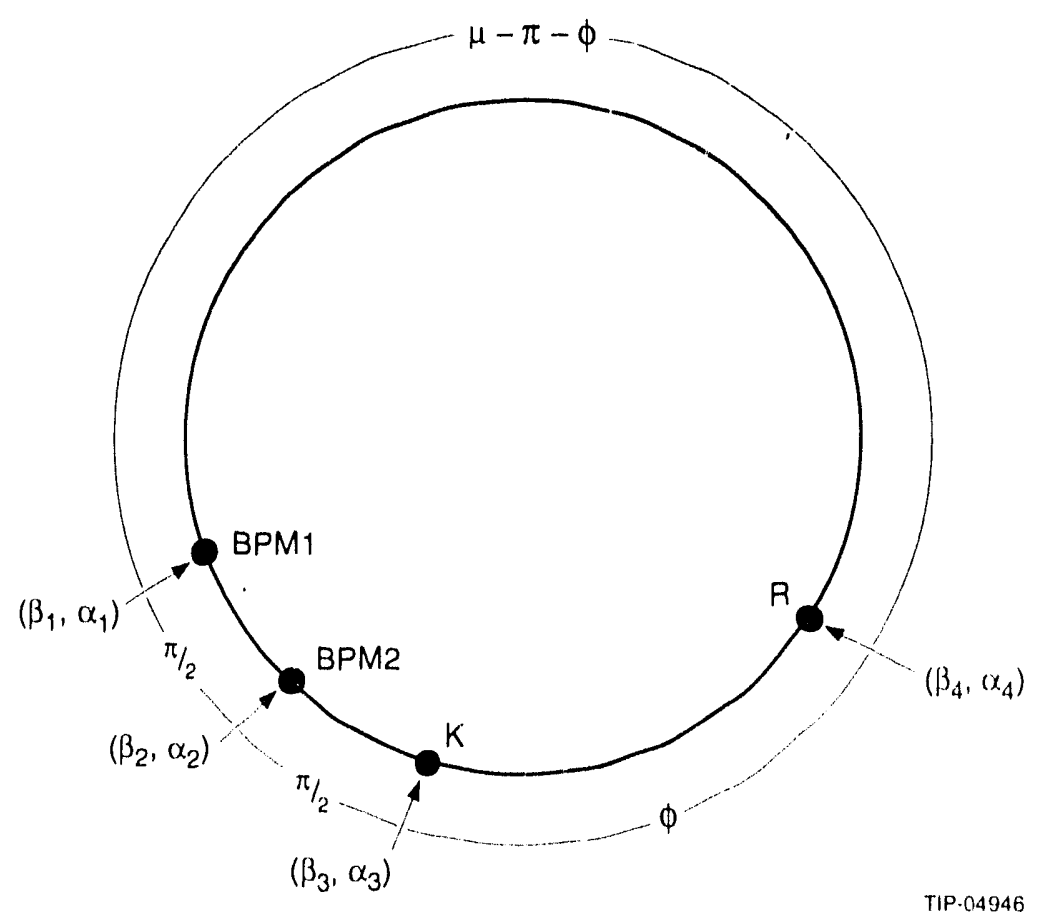

Figure 4. Collider model for the second scheme.

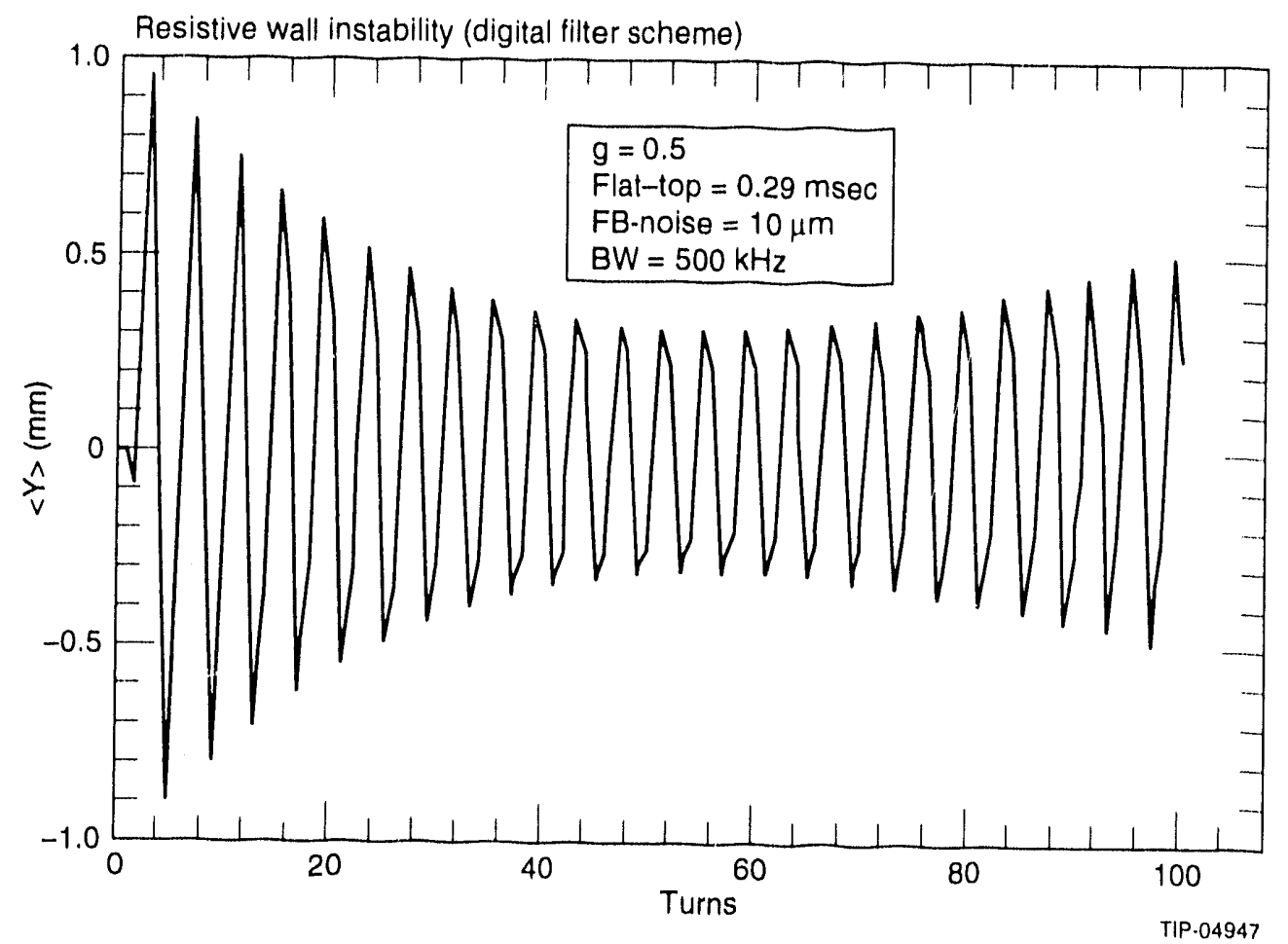

Figure 5. Average displacement seen by BPM2 


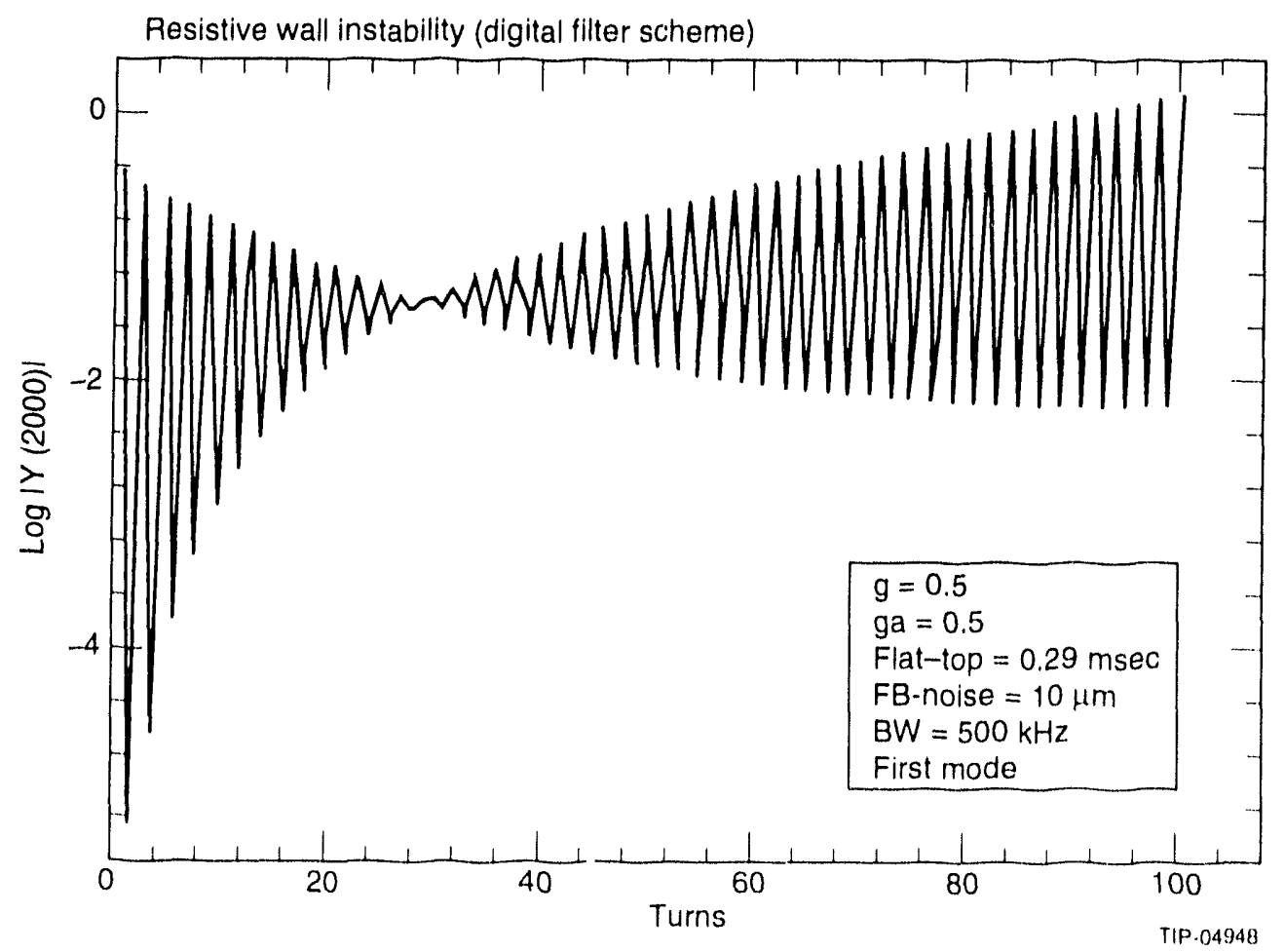

Figure (6. Behavior of the bunch number 2000 .

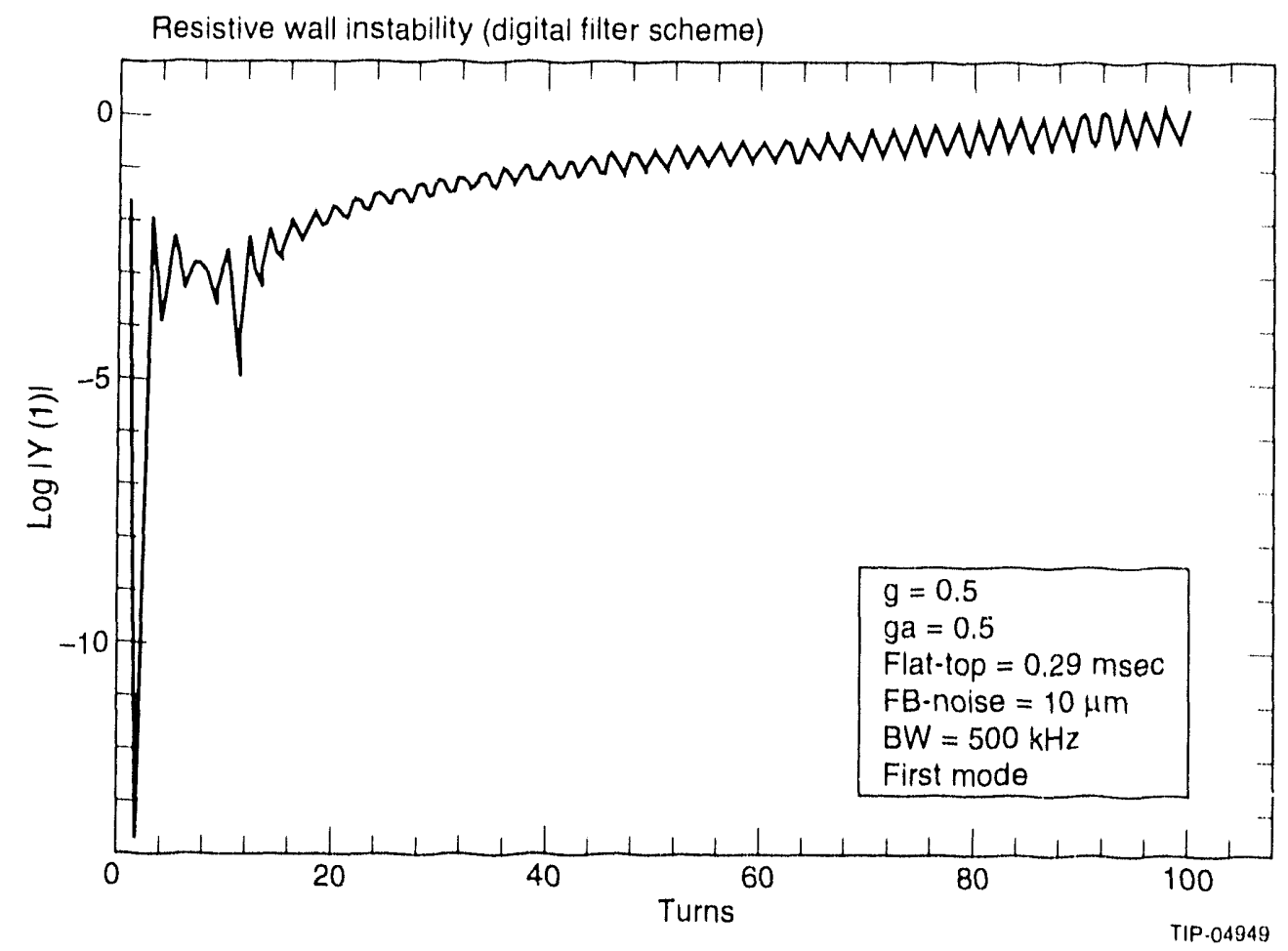

Pigure 7. Behavior of hunch number one 


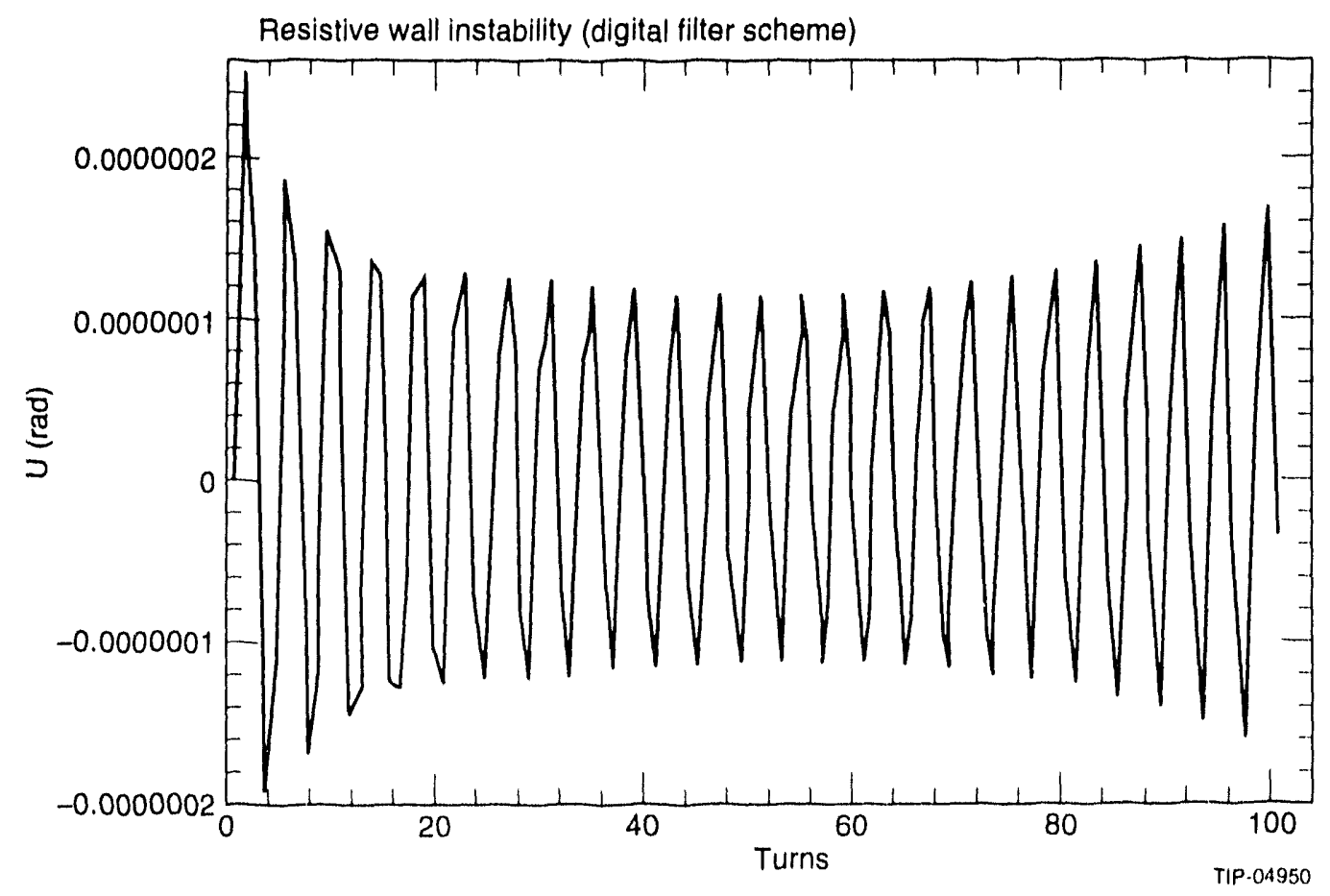

Figure 8. $U_{n}$ kick function.

\subsection{CONCLUSIONS}

Two feedback system schemes using a single kicker were studied from the point of view of controlling the resistive wall instability. The first scheme uses the subtraction of the previous amplitude recorded in the correction kick and is capable of controlling the resistive wall instability much better (the gain can be smaller) than the traditional same-tum correction kick. Its disadvantage is that it cannot kill the instability within two turns if the gain is equal to one. Its advantage is that it could be much less expensive than the two-BPM, two-kicker feedback system mentioned at the beginning. The second scheme uses a digital filter to provide a correction kick given by $\mathrm{Eq}_{1}$. (2) to E(1. (4). The digital filter scheme is not capable of controlling the resistive wall instability in the Collider. 


\section{ACKNOWLEDGEMENTS}

I wish to thank Drs. V. Parkhomchuk and V. Shiltsev for their valuable discussions, and Dr. R. Meinke for his support in the Collider group at the SSC laboratory. 


\section{REFERENCES}

1. S. Chen and G. López, SSCL-614, 1993.

2. G. López, E. Tsyganov, S. Chen, and R. Meinke, SSCL-N-815, 1993.

3. T. Tsyganov, G. Dugan, G. López, R. Meinke, W. Nexsen, and R. Talman, SSCLPreprint-202, 1993.

4. G. López, SSCL-625, 1993.

5. V. Parkhomchuk, internal communication, Maiy-August 1993.

6. V. Parkhomchuk and V. Shiltzev, internal seminary notes June 1993, also internal communication, 1993. 
